\title{
Automated Dimension Measurement System
}

\author{
Shubham Kakirde, Shubham Jain, Swaraj Kaondal, Reena Sonkusare, Rita Das
}

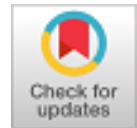

\begin{abstract}
In this fast-paced world, it is inevitable that the manual labor employed in industries will be replaced by their automated counterparts. There are a number of existing solutions which deal with object dimensions estimation but only a few of them are suitable for deployment in the industry. The reason being the trade-off between the cost, time for processing, accuracy and system complexity. The proposed system aims to automate the mentioned tasks with the help of a single camera and a line laser module for each conveyor belt setup using laser triangulation method to measure the height and edge detection algorithm for measuring the length and breadth of the object. The minimal use of equipment makes the system simple, power and time efficient. The proposed system has an average error of around $3 \%$ in the dimension estimation.
\end{abstract}

Keywords: Laser, Warehouses, Dimension Measurement, Automation.

\section{INTRODUCTION}

Efficient warehouse management is critical for an effective supply chain in the world where e-shopping is getting popular [1], [2]. A lot of warehouses even systems today employ manual labor for monotonous jobs like measuring dimensions of objects, scanning codes, etc. These systems are not only slow, but also not as cost effective as their automated counterparts [3], [4]. Also, in a post-Covid world, contact-less delivery will play a major role in warehouse systems, ensuring the safety of the customers. Many E-Commerce and E-Grocer Companies have their sales increased by $100 \%$ due to Covid19 [5]. There has been a drastic increase in the sales of packed foods, sanitizers and other household things. According to a report an E-Grocer company received 30,000 orders per day due to high requirement. Thus, as the demand rises, compromise in the services takes place. This may include wrong delivery of a product, delay in the shipment. Thus, a system is required that is faster, is contact less and involves fewer manual efforts. 3D

Manuscript received on April 02, 2021.

Revised Manuscript received on June 07, 2021.

Manuscript published on June 30, 2021.

* Correspondence Author

Shubham Kakirde*, Department of Electronics \& Telecommunications, Sardar Patel Institute of Technology, Mumbai (MH), India. Email: shubham.kakirde@spit.ac.in

Shubham Jain, Department of Electronics \& Telecommunications, Sardar Patel Institute of Technology, Mumbai (MH), India. Email: shubham.jain@spit.ac.in

Swaraj Kaondal, Department of Electronics \& Telecommunications, Sardar Patel Institute of Technology, Mumbai (MH), India. Email: swaraj.kaondal@spit.ac.in

Dr.Reena Kumbhare, Department of Electronic \& Telecommunications Sardar Patel Institute of Technology, Mumbai (MH), India. Email: reena kumbhare@spit.ac.in

Dr. Rita Das, Department of Electronics \& Telecommunications, Sardar Patel Institute of Technology, Mumbai (MH), India. Email: rita das@spit.ac.in

(C) The Authors. Published by Blue Eyes Intelligence Engineering and Sciences Publication (BEIESP). This is an open access article under the CC BY-NC-ND license (http://creativecommons.org/licenses/by-nc-nd/4.0/) reconstruction of objects using $2 \mathrm{D}$ images have been a great advancement in the field of computer vision. This can be done to analyze the object without any physical intervention in the process which makes it suitable for fragile objects. 3D reconstruction of objects also makes it easier for computational analysis and extracting the attributes of the object [6]-[8]. There are several solutions to solve the mentioned problem of estimating the dimensions of boxes. Each method employs a different set of hardware and different algorithmic process to achieve the same. Some focus extensively on the accuracy of the measurement while some focus on the operational speed and computational time. The power consumption varies based on the hardware employed. Height of a 3-D object can be measured with the help of a line laser and a camera. This is done with the help of laser triangulation method. The deflection in the line laser is checked by an algorithm to determine the shape of the surface of the object. Thus, this model works correctly for objects of any shape since the top surface is considered. Since, there is no estimation of the length and breadth of the objects, this model is not well suited for industrial applications where all the three dimensions of the object are required. The system that we are designing will consist of a single line laser and a camera module. This system focuses on utilizing as low power as possible because of a smaller number of components and high speed of operation which would be favorable for most of the industrial applications. The trade-off between the accuracy and the speed of operation is well suited for industrial needs. From algorithmic perspective, the only major operations would be masking the unwanted sections of the image and detecting the red laser line. This makes the process faster than the other observed methods which involve multiple lasers and cameras.

\section{METHODOLOGY}

In order to compute the three dimensions (length, breadth and the height) of an object, the initial step has to be calibrating the camera to map the pixel values to the real-world dimensions (in centimeters). This can be done by placing a line (of known length) of a distinct color on the ground level and detecting the number of pixels it takes in the image, this can be seen in the figure 5 . To identify the line on the surface we use edge detection algorithm [9]-[11]. The length and breadth that the camera will see depends upon the height of the box under the camera (closer objects appear larger). Hence, we first need to compute the height of the box. The displacement in the line laser projected on the box is directly proportional to the height of the box as shown in figure 4.

Once the displacement is obtained, the height of the box can be calculated. The camera being a point camera, there will be error introduced in the height of the box as shown in figure 2.

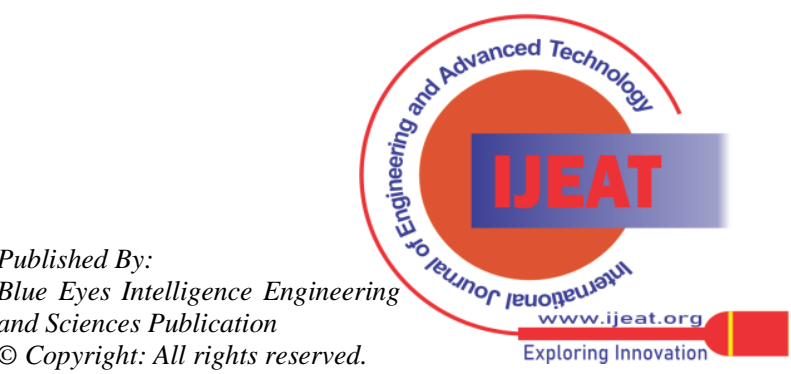




\section{Automated Dimension Measurement System}

This error can be eliminated by knowing how far the displaced laser line is from the center of the laser. Thus, the actual height of the box is obtained. The box is assumed to be in a certain color range. Hence, the unwanted colors in the image are masked so that only the box is visible. From the figure 9 the box length and breadth can be obtained in pixels using contour detection algorithms [12], [13]. If this pixel value is multiplied by the calibration factor, the projection of the dimension on the ground will be obtained as seen from figure 6 . The actual length and breadth of the box can be obtained by applying the principle of similarity of triangles.

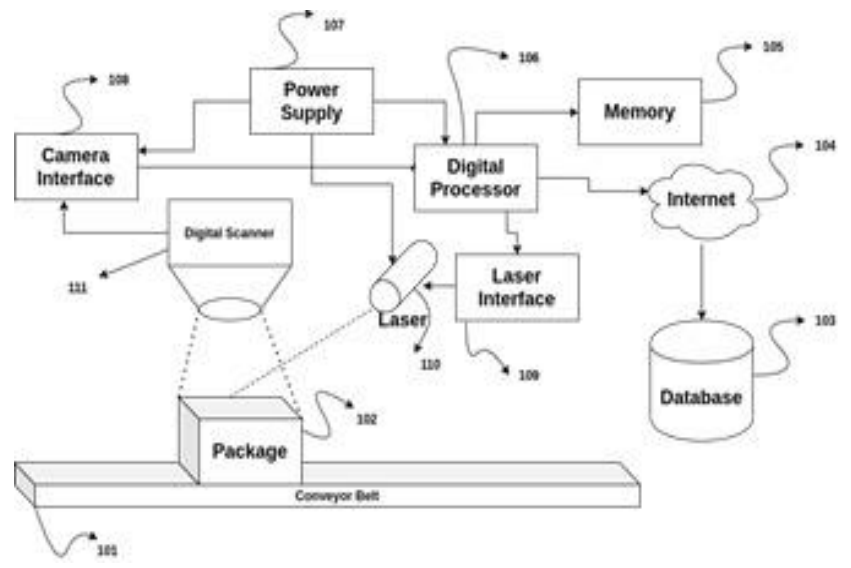

Figure 1: Block Diagram for the system

101: $\quad$ Conveyor belt over which objects pass

102: $\quad$ Package whose dimensions are to be calculated

103: Cloud database system where the information about objects is stored

104: The internet

105: Digital memory of the digital processor

106: Digital processor for processing the images

107: Power supply for the components

108: Digital interface between the digital scanner and the digital processor

109: Interface between the digital processor and the laser

110: Line laser for calculating the height of the objects

111: Digital scanner for scanning the objects on the conveyor belt

\section{MEASURING PROCEDURE}

\section{A. Eliminating Camera Error in Calculating Displacement}

Since the camera used is a pinhole camera, there is a error in calculating the distances in the plane perpendicular to the axis of the camera, when two objects are present in different planes and at different distances from the camera. While calculating the distance between the two-laser line, the distance recorded in the camera is not the required distance.

Figure 2 explains the error caused due to the fact that camera is a point camera. Hence, as objects get closer to the camera, their pixel coordinates start to vary based on their distance from the centre of the image.

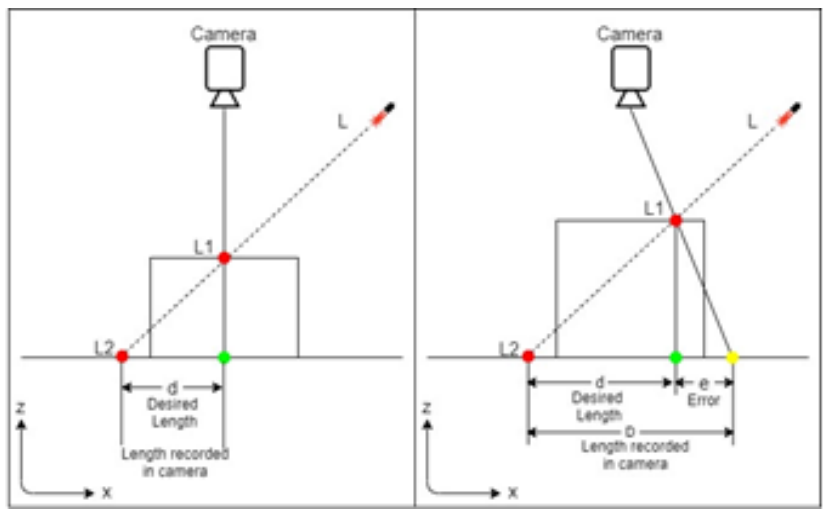

Figure 2: Concept of camera error

To get the desired length we need to calculate this error. To find the length ' $\mathrm{X}$ ' in figure 3 , we extract coordinates of the center of the image and the laser line on top of the box and subtract them. For a given camera height and the length recorded in the camera, the desired length can be calculated as follows.

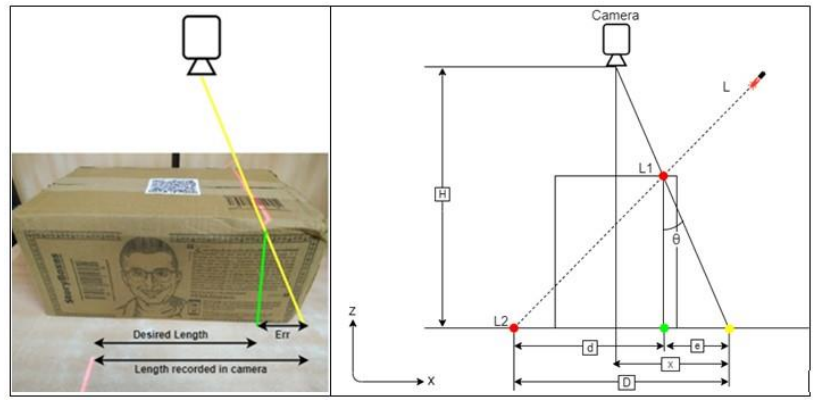

Figure 3: Correcting the camera error

Figure 3 explains the error caused due to the camera being a point camera. Here $\mathrm{H}$ is the height of the camera from ground level and $\mathrm{X}$ are the distance of the laser dot from the center of the image. Where,

$\theta$ : Angle formed by the vertical and the laser line on top of the box at the camera. $\varphi$ : Angle of inclination of laser. $\mathrm{d}$ :

Desired displacement.

D: Displacement observed by the camera.

$\mathrm{H}$ : Height of the camera from ground. e :

Error in the observed displacement.

$\mathrm{X}$ : Distance between the center of the camera and the laser line on the top of the box.

$\tan (\theta)=\frac{e * \tan (\phi)}{d}=\frac{X}{H}$

(1)

$$
\begin{gathered}
d *\left(1+\frac{X}{H * \tan (\phi)}\right)=D \\
d=\frac{D}{1+\frac{X}{H * \tan (\phi)}} \\
d=D-e=D-d * \frac{X}{H * \tan (\phi)}
\end{gathered}
$$

Published By:

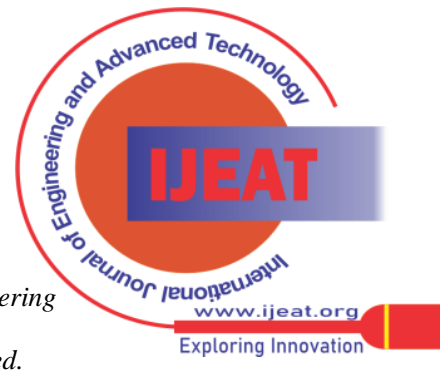




\section{B. Height Calculation}

As explained earlier, the height of the object will be proportional to the displacement in the laser. Also, from figure 4, it can be said that the height of the object is equal to the displacement in the laser light if the angle of projection of laser inclination is set to $45^{\circ}$. Implementing this system would give us the displacement of the laser line in number of pixels. The required result should be in units of distance like millimeters or centimeters.

The number of pixels which represent the displacement of the laser line will be linearly proportional to the actual height of the object. This means that to find the actual displacement, the pixel value has to be multiplied by a constant $(\mathrm{K})$. To find the value of $\mathrm{K}$, the camera is made to point to a surface from a height equal to the height of the camera in the system that is desired. Then, a line of known length is drawn on the surface and recorded by the camera as shown in figure 5 . After the length of the line is measured in pixels, the proportionality constant ' $\mathrm{K}$ ' can be calculated using the formula,

$$
K=\frac{d i s t \_i n \_c m}{\text { dist_in_pixels }}
$$

In figure 4, the top view explains what the setup will look like from top. The line laser is directed towards the box. Some part of the laser falls on the top surface of the box while some of it falls on the bottom surface. The side view explains the same setup seen from the side where the laser will be the top left of the triangle. The laser is fired such that it makes an angle of $\varphi$ with the vertical line in anti-clockwise direction.

This proportionality constant basically converts pixels to centimeters on the ground level. From the figure 4 it can be seen that the camera is able to capture the distance between the two lines of the laser. Let this length be "d" and let the height of the box be "h". Now using trigonometry, we can calculate the height, given the angle of inclination of laser light from the normal and the distance $\mathrm{d}$.

$$
h=\frac{d}{\tan (\phi)}
$$

Figure 5 explains the calibration of pixel values of camera reading to physical dimensions (in $\mathrm{cm}$ ).

The red line drawn on the white paper in figure 5 is of 20 $\mathrm{cm}$. The camera is held at a fixed height above the paper and the image is taken. This configuration will have a fixed $\mathrm{k}$. Note that every configuration depends on the distance of the camera from the ground and the angle at which the laser light plane is projected. In this case after applying some image processing techniques which will be discussed further, it is found that the length of the line is 232 pixels in figure 5 . This gives us a reference and the proportionality constant in this case is,

$$
\begin{gathered}
K=\frac{20}{232} \\
K=0.0862
\end{gathered}
$$

Now after finding the constant of proportionality, we can now find the height of any object under this configuration.

In equation 4 the quantity ' $D$ ' (displacement observed by camera) and ' $X$ ' (Distance between the center of the camera and the laser line on the top of the box) are recorded in pixels these quantities need to be multiplied by the constant $\mathrm{K}$ to convert in $\mathrm{cm}$.

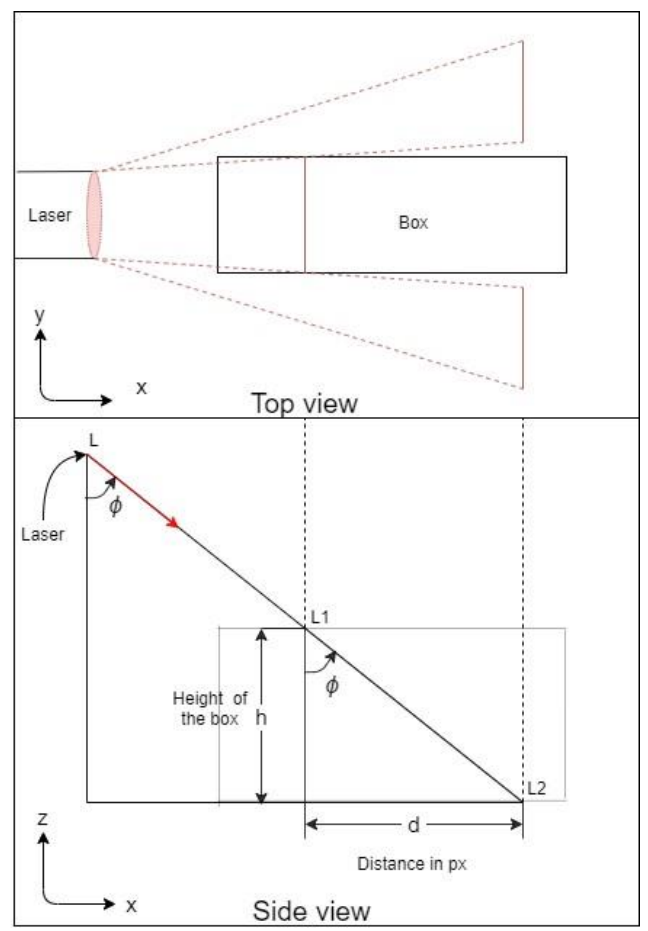

Figure 4: Top view and side view of the box under measurement

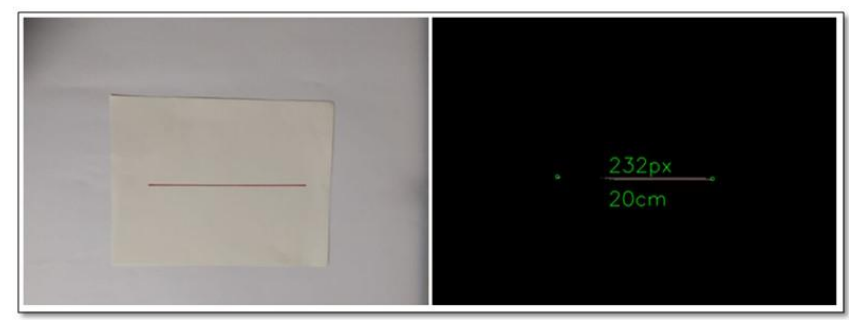

Figure 5: Calibration of the proportionality constant with the help of a $20 \mathrm{~cm}$ red line

Rewriting equation 4 ,

$$
d=\frac{K * D}{1+\frac{K * X}{H * \tan (\phi)}}
$$

Substituting equation 9 in equation 6 , we get,

$$
h=\frac{K * D}{\tan (\phi) *\left(1+\frac{K * X}{H * \tan (\phi)}\right)}
$$

\section{Length and Breadth Calculation}

For calculating the length and breadth we can directly use edge detection algorithms which will be discussed further. But again, the length and breadth of the box are in pixels and it needs to be converted into centimeters.

The calibration factor used to calculate the height of the box cannot be used in this case. The reason being that at different heights with respect to the base of the system the calibration factor changes. The calibration factor for the base can be calculated by drawing a line of known length and measuring it, but it is not possible to calculate it at every height. So to dynamically calculate the calibration factor at different heights we use the property of similarity of triangles in figure 6.

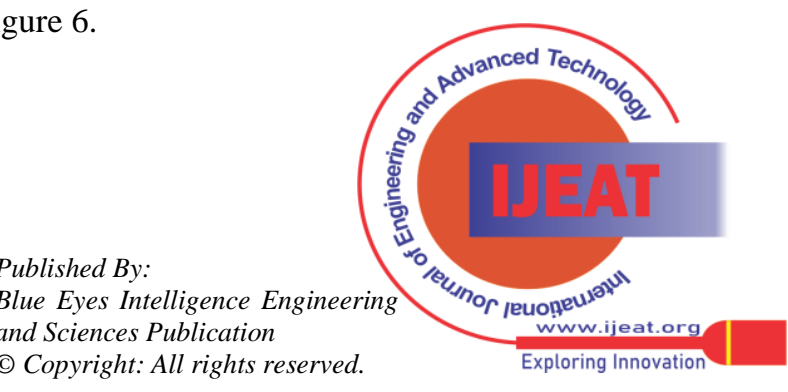




\section{Automated Dimension Measurement System}

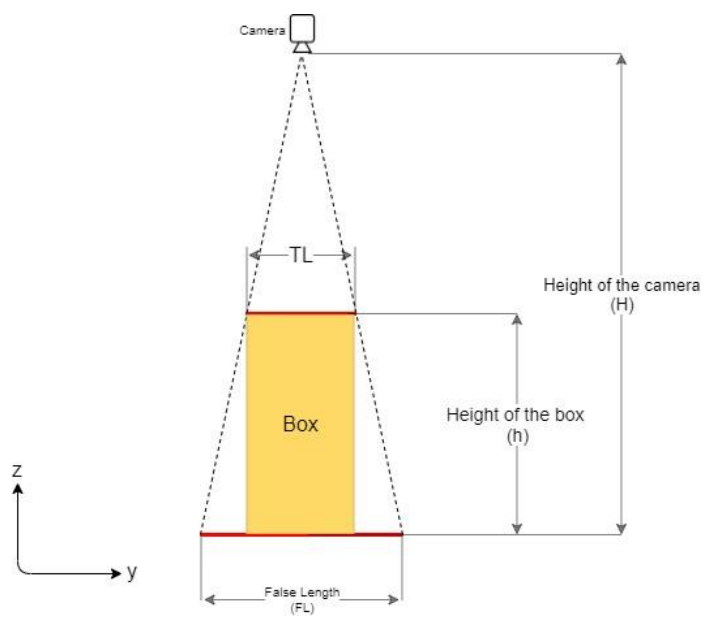

Figure 6: Length and Breadth calculation of the box

Figure 6 explains the concept of false length (projected length) and a method to calculate the desired length.

Now for a given configuration the height of the camera is fixed. Consider this height to be $\mathrm{H}$ refer 9 and the height of the box to be h, for simplicity lets calculate the length first. To calculate the length of the box in centimeters from the pixel values consider the height of the box to be approximately zero (h). So, the top face of the box will lie in the same plane as the $20 \mathrm{~cm}$ line used for calibration. Therefore, we can make use of the calibration factor $\mathrm{K}$ used to calculate the height and find the pseudo length. This is the length of the box at the height $\mathrm{H}$, then the length of the box at height $\mathrm{H}-\mathrm{h}$ will be given as,

$$
T L=F L * \frac{H-h}{H}
$$

where, $h$ : Height of the

box.

$\mathrm{H} \quad$ : Height of the camera from ground.

FL : False length recorded by the camera. TL

: Desired length.

Similarly, we can calculate the breadth of the box.

\section{ALGORITHMIC FLOW FOR ESTIMATION OF DIMENSIONS}

Under the given configuration we tried to measure the height of a box. The original image of the box can be seen in figure 7 . Figure 7 is the image of the box as taken from the camera without any processing.

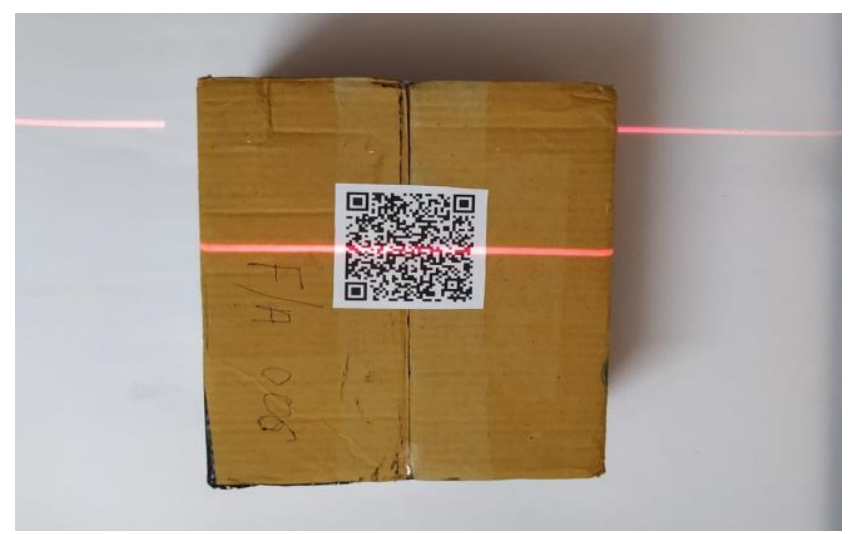

Figure 7: Original image of the box without any processing

\section{A. Height Estimation}

There are 4 major steps in the algorithm for height detection:

1) Masking: Masking is used to remove the part of the image that is not red. This is done to identify the lines made by the line laser and measure them accurately.

For applying the mask OpenCV library is used to perform the digital signal processing. First the mask is produced for both the upper and lower range of the red color and then it is combined. After that all the pixels which fall out of the range of the mask are made black. Leaving us with red lines of the laser. The output of masking can be observed in figure 8 .

2) Edge Detection: After the colors other than the laser light are masked from the image, the only part of the image remaining will be the three laser lines. In order to find the displacement in the laser light, we need to detect the edges of the laser lines. Before that can be done, the image has to be blurred in order to remove random noise that gets added to the image. The blurred image is then given to the edge detection algorithm so that the edges of the line laser can be detected. The difference in pixels between edges detected from the base of the laser line and the displaced laser line is detected. Thus, the displacement in line laser is measured. It then is converted to a dimension in centimeters.

3) Measurement: After applying the edge detection algorithm we get the coordinates of the points of the red laser line. Using these points, we find the two red laser lines in the image. The algorithm tries to group these points in two different sections and draws a straight line through each group. Then it calculates the midpoint of both the lines, joins them and passes the difference between the midpoints of the two lines further for calculating the height.

4) Calculation of height: The pixel value ' $D$ ' will be substituted in equation 10 to achieve the actual height of the box in centimeters. The distance between the two laser lines comes out to be $105 \mathrm{px}$, this distance is the length" $\mathrm{D}$ " in figure 4.

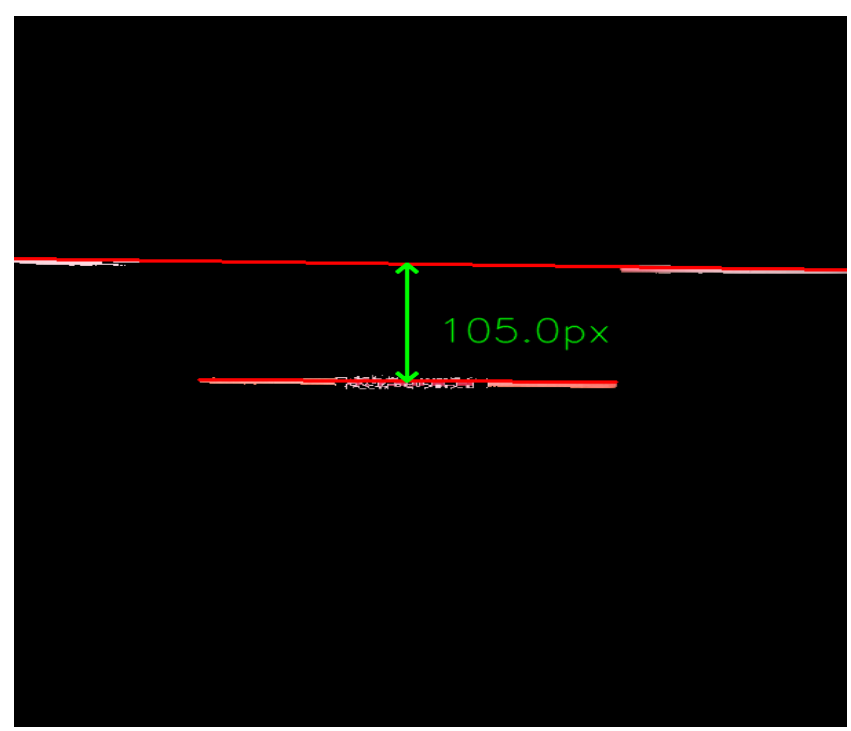

Figure 8: Distance between the laser line

Published By:

Blue Eyes Intelligence Engineering and Sciences Publication

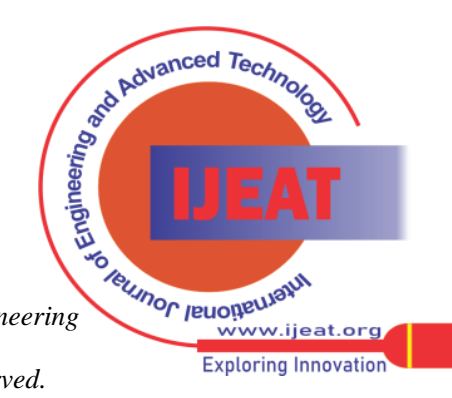




$$
h=0.0862 * \frac{105}{\tan (45) *\left(1+\frac{50 * 0.0862}{53}\right)}
$$

$h=8.37 \mathrm{~cm}$

\section{B. Calculation of Length And Breadth}

Calculation of length and breadth consists of four major steps:

1) Masking and Blurring: Masking simply means removing the part of the image that is not required. In the case of finding length and breadth of the box in the image, we will mask the box in the image. The resulting image will have background of 0 pixel value(black) and original box image will be retained. After masking, we perform blurring. The image is convoluted with a Gaussian filter. Gaussian Filter is a low pass filter that removes high frequency components.

2) Edge Detection: The output of the masking algorithm will produce an image where only the portion where box is present will be intact, while the other part of the image will be masked with black color. In order to find the edges of the box in the image, we need to apply an edge detection algorithm. The edge detection algorithm will detect the portion of the image as edges where there is an abrupt change in the pixel values. For the edge detection algorithm to work properly, initial blurring is required so that certain noise elements from the image get nullified and are not detected as edges. When the edges are detected, a bounding-rectangle algorithm will be run on the image where the detected edges are marked with a certain distinct colour (green in this case) so as to verify the correctness of the edge detection algorithm. The lengths of the edges can be obtained in pixels which then can be converted to real length and breadths in $\mathrm{cm}$.

3) Threshold: In threshold function the first argument is the source image, which should be a grayscale image. The second argument is the threshold value which is used to classify the pixel values. The third argument is the maximum value which is assigned to pixel values exceeding the threshold. Fourth argument is the type of thresholding we apply (here we use THRESH BINARY).

4) Bounding-Box: A bounding box is created around the box considering the threshold generated. Here bounding box will be rectangle in shape. (cv2.rectangle is used). Length and breadth detected is shown in pixels above the box.

The output of all these operations can be seen in fig. 9

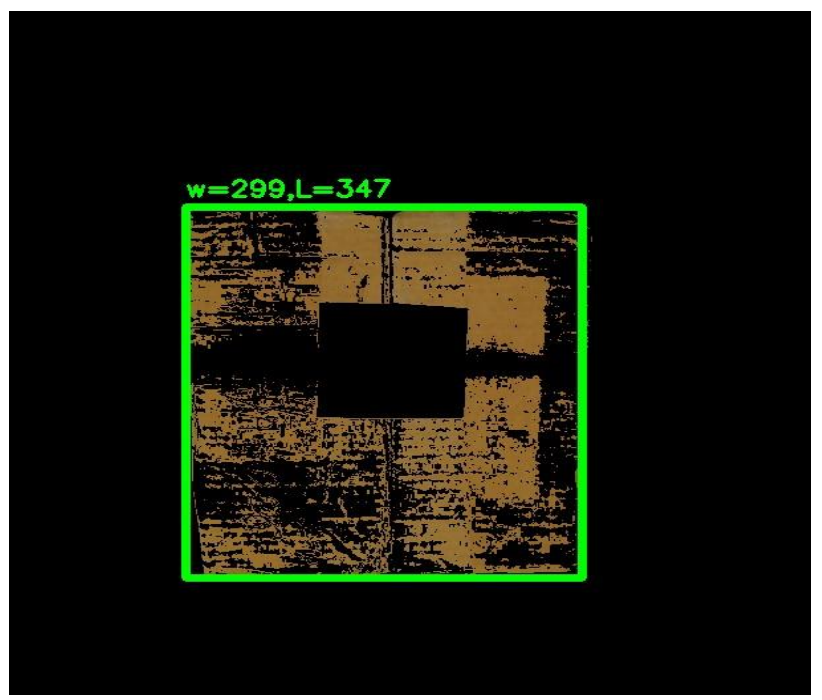

Figure 9: Length and Breadth of the box

Figure 9 shows the output of the box detection algorithm where all the colors outside the range of possible box colors are masked with black color, thus keeping the box visible.

This will ease the process of calculating the length and breadth of the box in pixels.

5) Calculation: After the boundary is defined, the edges are measured in pixels and then converted to centimeters using the methodology discussed previously. In this case the length and breadth in pixels are 347px and 299px respectively. To convert it into centimeters we use the given formula, 11

Table I is the tabulated form of the results that was produced after running the above algorithm on boxes of various sizes.

\section{RESULTS AND ANALYSIS}

\section{A. Results of the Proposed Methodology}

The results can be seen in table I. there can be minor errors in the dimension estimation. These errors can occur due to change in lighting conditions, the pattern on the object matches that of the laser, etc. The proposed methodology was tested to calculate the dimensions of 6 boxes.

Table 1 : Results Produced Using the Proposed Methodology

\begin{tabular}{|c|c|c|c|c|c|c|c|c|c|c|}
\hline \multirow{2}{*}{$\begin{array}{c}\text { Box } \\
\text { No. }\end{array}$} & \multicolumn{3}{|c|}{ Real dimensions } & \multicolumn{3}{|c|}{ Calculated dimensions } & \multirow[b]{2}{*}{ Length(\%) } & \multicolumn{2}{|c|}{ Error } & \multirow[b]{2}{*}{ Total(\%) } \\
\hline & Length(cm) & Breadth $(\mathrm{cm})$ & Height $(\mathrm{cm})$ & Length(cm) & Breadth $(\mathrm{cm})$ & Height(cm) & & Breadth(\%) & Height(\%) & \\
\hline 1 & 35.8 & 24 & 15.6 & 36.104 & 24.121 & 15.642 & 0.849 & 0.504 & 0.269 & 0.540 \\
\hline 2 & 35.8 & 15.6 & 24 & 37.043 & 16.567 & 23.402 & 3.472 & 6.198 & 2.491 & 4.054 \\
\hline 3 & 24 & 21.2 & 9.1 & 24.171 & 20.744 & 9.978 & 0.712 & 2.150 & 9.648 & 4.170 \\
\hline 4 & 21 & 9.5 & 24.5 & 22.151 & 9.661 & 23.648 & 5.480 & 1.694 & 3.477 & 3.551 \\
\hline 5 & 19.1 & 15.8 & 16 & 20.993 & 16.545 & 15.774 & 9.910 & 4.715 & 1.412 & 5.346 \\
\hline 6 & 20.3 & 16 & 16.5 & 20.178 & 15.942 & 16.263 & 0.600 & 0.362 & 1.436 & 0.799 \\
\hline Total & & & & & & & 3.504 & 2.604 & 3.122 & 3.077 \\
\hline
\end{tabular}

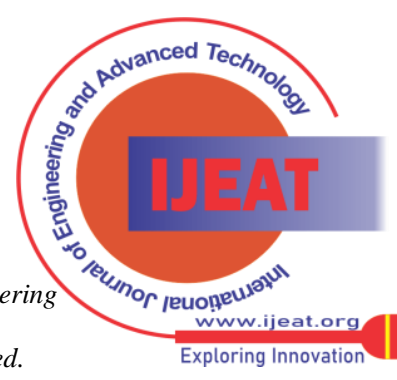


Automated Dimension Measurement System

Table 2: Comparative Analysis of Existing Methodologies.

\begin{tabular}{|c|c|c|c|c|c|c|c|c|c|}
\hline Parameter & & {$[14]$} & & {$[15]$} & & {$[16]$} & {$[17]$} & {$[18]$} & $\begin{array}{c}\text { Proposed } \\
\text { methodology }\end{array}$ \\
\hline Length(\%) & 1.1 & & 5 & & 8.5 & & 0.742 & 1.032 & 3.504 \\
\hline Breadth(\%) & 1.182 & & NA & & NA & & 0.05 & 4.1 & 2.604 \\
\hline Height(\%) & 1.502 & & NA & & NA & & NA & 3.6 & 3.122 \\
\hline Error(\%) & 1.261 & & 5 & & 8.5 & & 0.396 & 2.91 & 3.077 \\
\hline
\end{tabular}

As it can be seen from the above results, the error is close to $3 \%$. The error that can be seen in the cases is majorly due to the inaccurate laser angle and camera image quality.

\section{B. Comparison With Existing Methodologies}

Some of the existing methodologies are compared with ours to analyze the pros and cons of each one. There are some methodologies that employ multiple lasers and cameras or cameras with depth sensors, etc. Some measure the length of the objects based on how fast they are moving. Some of them only calculate single dimension of the objects.

Comments on existing methodology,

1) This methodology uses laser triangulation and deep learning methods for dimensions estimation. Two cameras and four lasers are required for the process. It produces very accurate results but the processing is very costly, leading to slower operations [14].

2) This methodology measures only the height of the object. The shape of deflected laser is considered so it can be used for curved surfaces as well. The camera error is not accounted for. This methodology uses only one camera and laser [15].

3) This method uses laser triangulation principle for height estimation. Only height of the object is calculated using this method. Camera error is not accounted for which leads to higher error. This methodology uses only one camera and laser [16].

4) The proposed methodology uses Computer Controlled Laser mounted Camera combo (CCLC) to measure the distance of the object from the camera and the laser triangulation method to measure the length and breadth of the object. The methodology uses two lasers and one camera, and the mean error is quite low [17].

5) To measure the height of the object, a camera with depth sensor is used. As the objects move over a conveyor belt (with known speed), the time that an object takes to cross the depth sensor is used to measure the length of the object. The error in the measurements is low. The error will depend upon the speed of the conveyor belt and the speed of the camera [18].

\section{CONCLUSION}

The proposed system can be used to estimate the dimensions of the objects very quickly and with minimal components as compared to other methods. The precision and speed of this methodology are well suited for industrial needs. Since only a single camera and laser are used, power consumption will be minimal. An error in measuring the
Laser Angle $\varphi$ can greatly affect the accuracy of the system. To minimize the errors that happen due to lighting conditions, the system can be enclosed in a casing with internal lighting. This system can also be integrated with an IOT based system to create a real time object tracking system. It can be easily integrated with any other automation system that makes use of object dimensions for efficient stacking of objects. Since the process of calculating the dimensions is contact-less it can be used to calculate the dimensions of fragile objects. This system will have high importance even in the post-covid world where contact-less operations are the key.

\section{REFERENCES}

1. Sneha Vishnu More," The study of Efficiency and Effectiveness of Warehouse Management in the context of Supply Chain Management," International Journal of Engineering Technology, Management and Applied Sciences, August 2016, volume 4, issue 8, ISSN 2349-4476.

2. V. Sivakumar, R. Ruthramathi," Challenges and Features of Warehousing Operations with Respect to Logistics Warehouse Companies in Chennai", International Journal of Innovative Technology and Exploring Engineering (IJITEE) ISSN: 2278-3075, Volume-9, Issue-1, November 2019.

3. Anas M. Atieh, Hazem Kaylani, Yousef Al-abdallat, Abeer Qaderi, Luma Ghoul, Lina Jaradat, Iman Hdairis, ” Performance Improvement of Inventory Management System Processes by an Automated Warehouse Management System",Procedia CIRP,Volume 41,2016,Pages 568572,ISSN 2212-8271.

4. Baker, Peter \& Halim, Zaheed. (2007). An exploration of warehouse automation implementations: Cost, service and flexibility issues. Supply Chain Management: An International Journal. 12. 129-138. 10.1108/13598540710737316.

5. Dr.Tareq N. Hashem," Examining the Influence of COVID 19 Pandemic in Changing Customers' Orientation towards E-Shopping", Online Published: July 14,2020,Modern Applied Science; Vol.14, No.8; 2020 ISSN:1913-1844, E-ISSN: 1913-1852

6. K. Wolff et al., "Point Cloud Noise and Outlier Removal for Image-Based 3D Reconstruction," 2016 Fourth International Conference on 3D Vision (3DV), Stanford, CA, 2016, pp. 118-127, doi: 10.1109/3DV.2016.20.

7. Remondino, F. and El-Hakim, S. (2006), Image-based 3D Modelling A Review. The Photogrammetric Record, 21: 269-291. doi:10.1111/j.14779730.2006.00383.x

8. Salih Burak Gokturk, Carlo Tomasi, Fahri Sur" uc“ u.(2007). Optical meth-" ods for remotely measuring objects. US patent US7310431B2. Canesta, Inc.

9. Wang, Z., Wang, W. The research on edge detection algorithm of lane. J Image Video Proc. 2018, 98 (2018). https://doi.org/10.1186/s13640-0180326-2

Published By:

Blue Eyes Intelligence Engineering and Sciences Publication

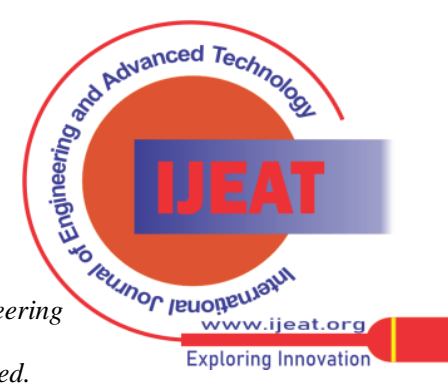


10. P. Dollar and C. L. Zitnick, "Fast Edge Detection Using Structured" Forests," in IEEE Transactions on Pattern Analysis and Machine Intelligence, vol. 37, no. 8, pp. 1558-1570, 1 Aug. 2015, doi: 10.1109/TPAMI.2014.2377715.

11. Maykel, Barbaro. (2015). ACCELERATING THE CANNY EDGE DETECTION ALGORITHM WITH CUDA/GPU.

12. Puri, Raghav Gupta, Archit. (2018). Contour, Shape \& Color Detection using OpenCV-Python.

13. Shanmugavadivu P., Kumar A. (2013) Boundary Detection of Objects in Digital Images Using Bit-Planes and Threshold Modified Canny Method. In: Prasath R., Kathirvalavakumar T. (eds) Mining Intelligence and Knowledge Exploration. Lecture Notes in Computer Science, vol 8284. Springer, Cham. https://doi.org/10.1007/978-3-319-03844-5 20

14. Peng, Gang-Ding Zhang, Yong Chen, Guanhua Zeng, Xinwu. (2019).Dimension Measurement and Key Point Detection of Boxes through Laser-Triangulation and Deep Learning-based Techniques. Applied Sciences. 10. 26. 10.3390/app10010026.

15. Wei Liu, Yongsheng Zhao, "Height Estimation of Target Objects Based on Structured Light", Advances in Multimedia, vol. 2018, Article ID 4189125, 9 pages, 2018 https://doi.org/10.1155/2018/4189125.

16. Wagh Rameshwar Ashok, M. S. Panse, Hemant Apte, "Laser Triangulation Based Object Height Measurement", INTERNATIONAL JOURNAL FOR RESEARCH IN EMERGING SCIENCE AND TECHNOLOGY, VOLUME-2, ISSUE-3, MARCH-2015, E-ISSN: 2349-7610.

17. Sharif, Muhammad HASSAN, M. KHAN, M. Raza, Mudassar KHAN, A.. (2013). Laser Guided Real Time Object Measurement and Distance Calculation System. Sindh Univ. Res. Jour. (Sci. Ser.). 45. 467475.

18. Park, Ho-Min Van Messem, Arnout De Neve, Wesley. (2018). BoxScan: An efficient and effective algorithm for box dimension measurement in conveyor systems using a single RGB-D camera

\section{AUTHORS PROFILE}

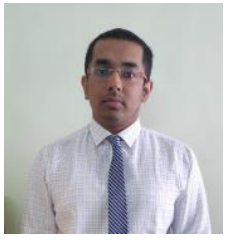

Shubham Kakirde, born in Mumbai, India in 1999 is currently a final year student in the Department of Electronics and Telecommunication Engineering, Sardar Patel Institute of Technology (S.P.I.T), Mumbai. Shubham Kakirde has always been an enthusiast geek with a "Never Stop Learning" motto. His passion lies in the field of IOT and Web Technologies.

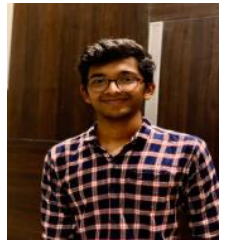

Shubham Jain, born in Mumbai, India in 1999 is currently a final year student in the Department of Electronics and Telecommunication Engineering, Sardar Patel Institute of Technology (S.P.I.T), Mumbai. Shubham Jain always believes in "learning by doing". His passion lies in the field of Web Technologies and Machine Learning

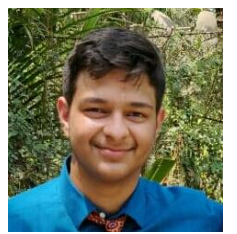

Swaraj Kaondal, born in Mumbai, India in 1999 is currently a final year student in the Department of Electronics and Telecommunication Engineering, Sardar Patel Institute of Technology (S.P.I.T), Mumbai. Swaraj Kaondal is an honest and hardworking individual with smart working ethics. His fields of interest are IOT, automation and AI.

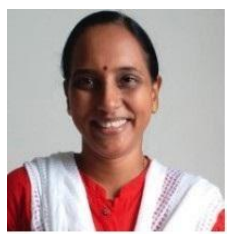

Reena Sonkusare, received her Ph.D. degree from the University of Mumbai, India in 2018. She has published more than 50 papers in various national and international conferences and published papers in international journals like Elsevier Microelectronics Journal, Springer Analog Integrated Circuits and Signal Processing, VLSI Circuits and Systems Letter IEEE computer society etc. She is currently a Associate professor and Head of Electronics and Telecommunication Engineering department at the Sardar Patel Institute of Technology, University of Mumbai, India. Her special fields of interest include nanotechnology, device modelling, RF and Mixed signal circuit design.

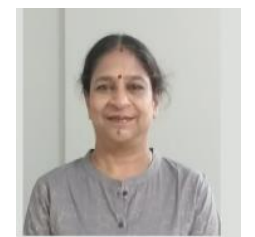

Rita Das, received her Ph.D. degree from I.I.T., Kanpur, India in 1992. She has published research papers in international journals like Physical Review B, JMMM and JMS.She is currently an Associate Professor, Head of Applied Sciences and Humanities and Dean of Students Affairs at Bhartiya Vidya Bhavan's Sardar Patel Institute of Technology, University of Mumbai, India. Her special field of interest is magnetic materials.

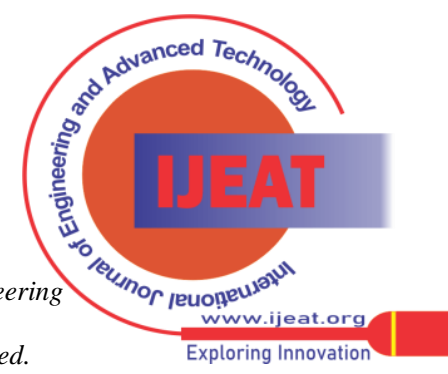

